\title{
Análisis de la comunicación social en el Homeschooling: una revisión panorámica
}

\author{
Evelyn Díaz-Hernández a, *, Milena García-García a \\ a Escuela de Medicina y Ciencias de la Salud, Universidad del Rosario, Colombia
}

\section{RESUMEN}

En el siglo XXI, las transformaciones al sistema social han generado alternativas educativas en las que el fonoaudiólogo adopta un papel importante, por ser un promotor de habilidades comunicativas. La educación en casa es una opción, sin embargo, existen controversias sobre su impacto en el desarrollo de habilidades sociales en los niños. Este artículo realiza una revisión panorámica de la literatura que explora la relación entre el Homeschooling y el desarrollo de la comunicación social. Se incluyen estudios publicados entre el 2000 y el 2020, en idioma español o inglés, con resumen y texto completo. Se excluyeron aquellos que incluían participantes con discapacidad cognitiva o trastornos del aprendizaje. En total de 328 artículos encontrados, sólo 9 cumplieron con los criterios de inclusión. Los documentos seleccionados se analizaron de acuerdo con los componentes de la comunicación social: interacción, cognición social y pragmática en comunicación verbal y no verbal. Existen discrepancias entre los resultados. Algunos autores afirman que las habilidades de comunicación social de los niños que aprenden en sus casas son mejores que las de los niños que van a la escuela, pero, en otros casos, estos solo cumplen con estándares esperados para su edad. Se identificó como determinante el papel de los padres o educadores. Sobre habilidades pragmáticas no se encontraron estudios. En conclusión, la limitación en el número de artículos y la heterogeneidad en las metodologías presentadas no permiten generalizar los resultados a la población. Es evidente la necesidad de realizar estudios en esta área, especialmente en este momento de confinamiento debido a la pandemia por el COVID-19.

\section{Social communication analysis in Homeschooling: a scoping review}

\begin{abstract}
In the 21 st century, transformations to the social system have generated educational alternatives in which the speech language pathologist as a promoter of communicative skills takes an important role. Home education is an option, however, there are controversies about its impact on children's social skills development. This article is a scoping review that explores the relationship between Homeschooling and the social communication development. Studies published between 2000 and 2020 , Spanish or English, with summary and full text are included. Those that included participants with cognitive disabilities or learning disorders were excluded. In total of 328 items found, only 9 met the inclusion criteria. The selected documents were analyzed according to the components of social communication: interaction, social and pragmatic cognition in verbal and nonverbal communication of children. There are discrepancies between the results. Some authors claim that social communication skills of children in homeschooling are better than those of children going to school, but in other cases, they meet expected standards for their age. The role of parents or educators was identified as decisive. No studies were found in pragmatic skills. In conclusion, the limitation on the number of articles and heterogeneity in the methodologies presented does not allow the results to be generalized to the population. The need for studies in this area is evident, especially at this time of confinement due to the COVID-19 pandemic.
\end{abstract}

\footnotetext{
* Autor/a correspondiente: Evelyn Díaz-Hernández

Email:evelyn.diaz@urosario.edu.co
}

\section{Palabras clave: Educación personalizada; Comunicación social; Interacción social; Fonoaudiología escolar; Educación en casa}

\section{Keywords: Personalized education; Social communication; Social interaction; School speech therapy; Homeschooling}

Recibido: 22-02-2021

Aceptado: 13-09-2021

Publicado: 13-11-2021 


\section{INTRODUCCIÓN}

En el proceso de enseñanza-aprendizaje se considera la escuela como el elemento esencial para que el niño apropie las habilidades cognitivas, lingüísticas y sociales desde un plano interindividual a uno intraindividual (Vigotsky, 1977). La educación se convierte en un mediador social (Álvarez \& del Río, 1990) y se percibe al infante como un ente «social, activo, protagonista y producto de múltiples interrelaciones sociales» (Chaves Salas, 2001). Así, se espera que el niño alcance un nivel sofisticado de competencia social que será un organizador central en el desarrollo (Guralnick, 1990). Además, implicará que sea capaz de responder de manera flexible a nuevos desafíos sociales a partir de sus experiencias previas (Semrud-Clikeman, 2007). Entonces, la comunicación social tiene un papel importante (Orpinas, 2010; SemrudClikeman, 2007; Topping et al., 2000) para que el niño consiga objetivos sociales en contextos específicos (Ford, 1982).

Inicialmente, Hovland (1948) define la comunicación social como «el proceso en el que el individuo transmite símbolos para modificar la conducta de otros individuos» y describe cuatro factores importantes: el comunicador quien transmite información, el estímulo transmitido por el comunicador, el individuo quien responde a la comunicación, y la respuesta al momento de comunicarse. Posteriormente, Adams (2005) la concibe como «la emergencia sinérgica entre la interacción social, cognición social, pragmática (aspectos verbales y no verbales) y el procesamiento del lenguaje (receptivo y expresivo)». Luego, Garcia-Winner (2011) la describe como la capacidad de «adaptar el comportamiento de manera efectiva en función de la situación y de lo que se sabe sobre las personas para que el emisor pueda reaccionar y responder de la manera en la que se espera». Después Izaryk \& Skarakis-Doyle (2017) la entienden como sinónimo de las habilidades pragmáticas. Finalmente, Denworth (2018) plantea que es un proceso intrínsecamente social, pues requiere la capacidad de compartir, comprender y responder a lo que otros sienten o dicen.

En esta revisión se adoptará la perspectiva de Adams (2005) porque este autor elabora un modelo para describir a la comunicación social desde cada uno de sus componentes, que en la actualidad es un referente para la American Speech- LanguageHearing Association ASHA. Por ello, a continuación, se detallará cada componente propuesto en esta perspectiva.

La interacción social puede ser comprendida como el intercambio de información entre dos o más participantes situados en contextos sociales. Mediante este proceso los sujetos adquieren capacidad reflexiva para verse a sí mismos y dar tanto forma como sentido a la realidad social que los rodea (Rizo García, 2006). Además, la interacción social motiva e incita a la producción del lenguaje en cualquiera de sus modalidades (gestual, verbal o no verbal) con un propósito determinado.

Por su parte, la cognición social se define como un proceso neurobiológico, psicológico y social (Bases cognitivas de la conducta social humana, 2003). Es responsable de percibir, reconocer y evaluar los eventos sociales para construir una representación del ambiente de interacción de los individuos y generar el comportamiento social o la respuesta más adecuada, según la situación particular (Adolphs, 2001). Además, se relaciona con la percepción social, que es el estadio inicial que evalúa las intenciones de las personas a través de la conducta, el lenguaje no verbal y el estilo atribucional (Pelphrey et al., 2004).

La pragmática (verbal y no verbal) se relaciona con la habilidad para seguir reglas que se ajusten a la cultura en determinadas situaciones comunicativas (American Speech-Language-Hearing Association [ASHA], s. f.-a). También se vincula con el uso de los recursos para transmitir significados relacionados a los contextos de uso cotidiano (Serra, 2013; Stainton, 2000). Puede ser dividida en: a) intenciones comunicativas expresadas directa o indirectamente, b) presuposición que implica la capacidad de hacer suposiciones sobre las necesidades de información del interlocutor, c) habilidades de procesamiento de información y el estatus social y d) habilidades de manejo del discurso que involucran la capacidad de usar estrategias apropiadas de iniciación, mantenimiento y terminación de temas (Landa, 2005).

Finalmente, el procesamiento del lenguaje receptivo y expresivo incluye la forma en la cual es recibida y analizada la información a través de las vías visual y/o auditiva. También, se relaciona con la manera y la capacidad de los seres humanos para comunicarse a través del lenguaje hablado y/o escrito. El procesamiento lingüístico incluye el desarrollo de las habilidades a nivel fonológico, morfológico, sintáctico y semántico.

De esta forma, la comunicación social involucra la capacidad de variar el estilo de habla según el contexto, considerar la perspectiva de los interlocutores, comprender y usar apropiadamente las reglas para la comunicación verbal y no verbal y utilizar los aspectos estructurales del lenguaje (American Speech-Language-Hearing Association [ASHA], s. f.-b).

Respecto al desarrollo comunicativo del niño esta perspectiva sostiene que la comunicación se adquiere al estar expuesto a una serie de actividades eminentemente sociales (Adams, 2005). Durante el proceso de desarrollo comunicativo del niño, la comunicación social le permitirá lograr objetivos funcionales a 
través de la interacción con otros (Acuña \& Sentis, 2004; Diaz Favier, 2004). Por lo anterior, la escuela adquiere importancia, ya que proporciona un contexto en el cual se desarrollan aspectos fundamentales de la personalidad, incluida la cooperación con sus compañeros y la adquisición de habilidades sociales (Flores, 2010).

En la edad preescolar gran parte de las intervenciones de los niños se refieren al presente, sus intercambios comunicativos son breves y no van más allá de unos cuantos turnos de conversación (Owens, 2011). Durante los siguientes años, se convierten en interlocutores conversacionales auténticos, ya que amplían su capacidad para adoptar la perspectiva de los demás participantes y son más conscientes de los roles sociales a medida que se van haciendo mayores (Shatz \& Gelman, 1973). En este punto, la participación de forma desplegada y profunda en el juego temático de roles sociales permite que los niños puedan pasar con éxito de la etapa preescolar a la escolar (Bredikyte \& Hakkarainen, 2007; Solovieva \& Quintanar, 2012).

En el enfoque histórico-cultural y la teoría de la actividad, el juego temático de roles es una forma particular de adquisición de la experiencia social y cultural (Vigotsky, 1984). Por esto se dice que el juego de roles tiene un origen social (Elkonin, 1980). Al principio, la actividad lúdica se presenta a partir de la acumulación de las intenciones del adulto, quien propone el juego al niño (Solovieva \& Quintanar, 2012). Aquí, el juego es atractivo por medio de la actividad conjunta que se comparte. El niño interactúa con objetos, considerando propósitos específicos e intencionales y después usa sustitutos de objetos. Luego, empieza a influir en su conducta y en la de los demás a partir de su propio lenguaje verbal externo. La motivación que surge en este proceso es importante para el desarrollo de la personalidad porque le permite al niño aprender a comportarse de acuerdo con la situación. Así, se origina la orientación hacia las normas sociales.

A medida que el niño crece, amplía su red social, modifica paulatinamente su autoimagen y su autoestima y se hace más conscientes de las expectativas sociales (Cicchetti \& Carlson, 1989). Entonces, su lenguaje refleja una red social más amplia y una nueva necesidad de comunicación (Bretherton \& Beegly, 1982), logrando relacionarse con otras personas en diferentes contextos o situaciones (Dongil-Collado \& Cano-Vindel, 2014).

Las dificultades que pueden manifestar los niños en esta esfera se pueden traducir en una incapacidad para expresar necesidades y deseos durante las interacciones con los pares, problemas para comprender la perspectiva de otra persona, dificultad para tomar turnos y mantener el tópico conversacional. Todo lo anterior puede tener consecuencias sociales negativas en la interacción social del niño, lo que puede provocar el rechazo de los pares (Hadley \& Schuele, 1995).

En relación con lo anterior, se discuten los efectos de los sistemas educativos en las habilidades sociales, incluida la comunicación. Por ejemplo, la educación en casa o Homeschooling es una opción educativa adoptada por quienes deciden educar a sus hijos en el hogar al margen del sistema educativo tradicional (Red En Familia, 2012). En la década de 1960 se propone que la escuela es poco favorable para la educación. Por ello, se origina la modalidad educativa Homeschooling en Estados Unidos, Canadá, Australia y el Reino Unido (Cabo-González, 2012). Este es un fenómeno en evolución, que genera diferentes posturas y actitudes que varían entre los países. Por ello, se producen cambios en el marco legal que lo reglamenta (Luebke, 1999). Además, esta modalidad educativa exhibe tanto ventajas como desventajas.

Entre las ventajas destaca el uso de métodos y herramientas novedosas que incluyen recursos de aprendizaje diseñados para la supervisión de los padres, dejando de lado el uso de pizarras o las calificaciones tradicionales (Red En Familia, 2012). Se produce una enseñanza más flexible que se adapta a las necesidades del menor y estimula aspectos como la autogestión y la metacognición (Benabent, 2015). Las actividades pueden ser llevadas a cabo en cualquier horario durante el día, ya que no requieren de una exigencia horaria. Además, los currículos ofrecen oportunidades para fortalecer la relación entre padres e hijos, de manera que su comunicación se vuelve más fluida y próxima (Cabo-González, 2012). Sin embargo, sus padres necesitan involucrarse activamente en el proceso con un entrenamiento previo para evitar perder la objetividad de la información que se proporciona a los niños (Run, 2016). Para esto, existen diversos programas de formación y cooperación a los padres que desarrolla competencias en diferentes áreas del conocimiento (Valle-Aparicio, 2012).

Algunas desventajas son que los niños expuestos a la educación en casa podrían tener oportunidades reducidas de crear o generar vínculos sociales con otras personas del entorno, especialmente en espacios para descansar o para recrearse. Ello debido a que en dichos espacios los niños deben interactuar con pares, lo que les permite el desarrollo de habilidades sociales (Pereira Del Prette \& Del Prette, 2003). Lebeda (2007) menciona que los niños escolarizado en el modelo Homeschooling presentan dificultad para socializar, debido a que el hogar los restringe a un solo espacio o contexto. Se sabe que para la interacción exitosa es necesarios que las personas tengan experiencias en tres contextos: hogar, escuela y establecimiento de relaciones sociales. En 
consecuencia, el Homeschooling limita a los niños la posibilidad de experimentar interacciones en diferentes contextos de socialización.

De acuerdo a lo expuesto previamente, es posible afirmar que no existe consenso sobre el impacto que tiene el Homeschooling en el desarrollo de las habilidades sociales de los niños. Por ello, el objetivo de este estudio es describir la relación entre la educación del hogar y el desarrollo de la comunicación social de los niños que participan de este modelo educativo. Se retoma el concepto de comunicación social y se explora cada uno de los componentes propuestos por Adams (2005) y la (American Speech-LanguageHearing Association [ASHA], s. f.-a) para orientar el análisis de la información.

\section{MÉTODO}

Se plantea una revisión panorámica teniendo en cuenta los criterios propuestos por (Arksey \& O’Malley, 2005). Se presenta un relato narrativo de la literatura existente, sin evaluar la calidad de la evidencia encontrada ni la heterogeneidad de los artículos reportados. Tampoco se discuten las fuentes de error. Por este motivo, no se pueden generalizar los hallazgos de los estudios particulares.

\section{Estrategia de búsqueda}

La búsqueda de información se elaboró inicialmente a partir de la pregunta PICO (Población, Intervención, Comparación y Resultados), que permite la búsqueda de evidencias necesarias para la investigación. A partir de esta pregunta, se generaron las palabras clave y la llave de búsqueda: ("Homeschooling" OR "home schooling" OR "home education" OR "homeschool" OR "unschooling”) AND ("pragmatics" OR "social interaction" OR "social development" OR "socialization" OR “communication" OR language").

La pesquisa se realizó en las bases de datos 1 findr free edition, Communication \& Mass Media Complete, Scopus, Web of Science, Education Database, ERIC y Academic Search
Complete, que incorporan la mayoría de las publicaciones disponibles en el área de educación.

Se incluyeron los resúmenes o textos completos de artículos originales, ya sea escritos en inglés o en español. Los artículos debían ser producto de investigaciones realizadas entre el primero de enero del año 2000 hasta el último día de febrero del año 2020. Este rango de fechas fue seleccionado teniendo en cuenta que la definición de los componentes de comunicación social es propuesta por Adams en el año 2005 y el objetivo de la revisión era buscar la información más reciente sobre dicha propuesta.

Se excluyeron los estudios realizados en población con discapacidad intelectual, dificultades en el desarrollo, trastornos del aprendizaje o de origen neurológico o psiquiátrico. Para estas poblaciones se realizan adaptaciones educativas que en algunos casos se incorporan a las políticas de inclusión escolar, por lo que no serían generalizables al Homeschooling. Además, el objetivo de este estudio es evaluar el impacto en población normotípica.

\section{Selección de artículos}

Se recuperó un total de 328 documentos con la llave de búsqueda en las diferentes bases de datos. Se incluyeron solamente 37 artículos, que en el título y/o en el resumen mostraban algún tipo de relación con el objetivo de la revisión. Los autores de este estudio analizaron los resúmenes para eliminar aquellos que no se ajustaban a los criterios. Un total de 21 artículos con texto completo fueron recuperados. De ellos, sólo nueve artículos cumplieron los criterios de inclusión para la investigación. Los doce restantes fueron excluidos por las siguientes razones: a) existía ausencia de relación de los resultados con el objetivo de la revisión, b) eran recopilación de evidencia a través de revisiones sistemáticas, y c) incluían a población con discapacidad intelectual.

En la figura 1 se describe el proceso de búsqueda y selección de los artículos. 

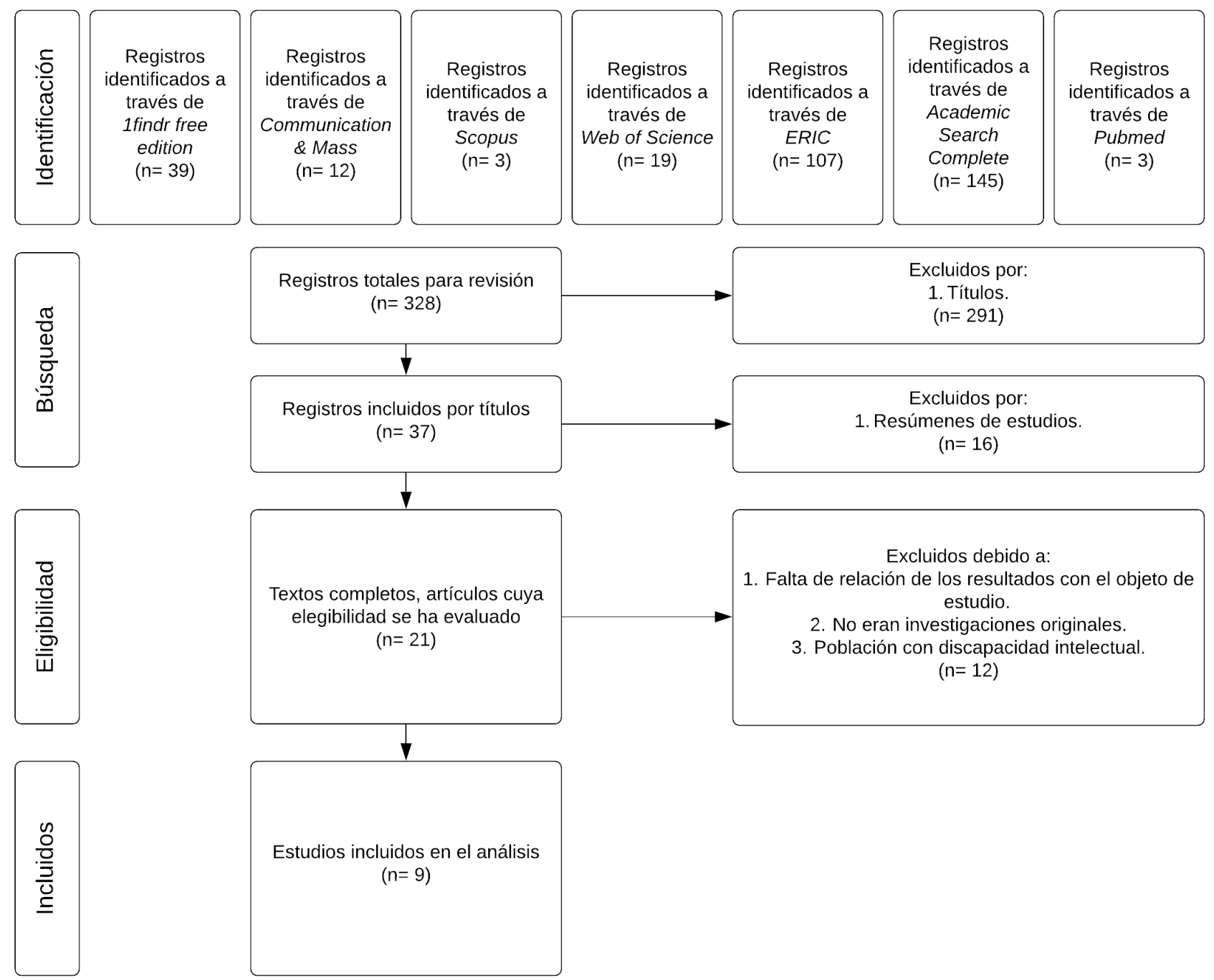

Figura 1. Desarrollo del proceso de búsqueda de artículos.

\section{Análisis de datos}

Se diseñaron tres matrices en el programa Excel de (Microsoft $\AA$ ) para analizar los artículos. En primer lugar, la matriz de selección de artículos, de acuerdo con la base de datos en la que se evaluaba si cumplía con los criterios de inclusión. En segundo lugar, la base de análisis de textos completos, según título, objetivo, participantes, tipo de estudio, diseño metodológico y resultados. La tercera matriz contenía los componentes de la comunicación social (Adams, 2005), que incluye aspectos relacionados con la interacción social, cognición social, el procesamiento del lenguaje expresivo y comprensivo y la pragmática en las modalidades de comunicación verbal y no verbal. En todos los casos se hizo una síntesis narrativa de los resultados a partir de las categorías de análisis propuestas.

\section{RESULTADOS}

Se seleccionaron nueve artículos que cumplían con los criterios de inclusión. La tabla 1 muestra un resumen de los artículos que incluye la siguiente información: país, autor (es), título, objetivo, metodología, participantes y resultados (Anexo). Se encontraron cuatro estudios de carácter cualitativo, dos de carácter cuantitativo, dos mixtos con predominio cualitativo y uno mixto sin predominio de ningún carácter. Las publicaciones abordaron 
temáticas relacionadas con la educación en el hogar y su relación con el desarrollo de la comunicación social. La mayoría de los estudios informaron un impacto positivo de la educación en el hogar en términos de habilidades de interacción y cognición social.

Los participantes de los estudios eran niños educados en el hogar, niños pertenecientes a escuelas públicas de tercer a sexto grado y niños que recibían educación privada. También se incluyeron, en algunos casos, como participantes a los padres de los niños con educación en el hogar y educación pública. Los países en donde se desarrollaron estas investigaciones son: Estados Unidos, Noruega, Indonesia, Israel y Chile. La muestra más pequeña fue de 16 participantes pertenecientes al Homeschooling y la muestra más grande fue de 564 padres noruegos con hijos que asistían a la escuela, que era obligatoria.

Los instrumentos de evaluación que medían el impacto en la comunicación social fueron: cuestionarios, escalas estandarizadas, entrevistas, encuestas, cuestionarios de autopercepción, listas de verificación del comportamiento infantil y escenarios hipotéticos. Este último instrumento expone situaciones ficticias que representan la victimización física o social. A través de estas situaciones, se busca que identificar a participantes que han vivido situaciones similares. A ellos se les consulta la frecuencia y se les solicita una opinión personal frente a este escenario hipotético (Reavis \& Zakriski, 2005).

A continuación, se describen y organizan los resultados de acuerdo con cada componente de la comunicación social.

\section{Interacción social y establecimiento de vínculos afectivos}

La interacción social de los niños educados en el hogar se asoció principalmente a las actividades que podían desarrollar (Aliaga Castillo, 2017; Guterman \& Neuman, 2017). Generalmente, los niños tienen diferentes clases o talleres deportivos y artísticos, que se suman a actividades sociales en cada comunidad (Aliaga Castillo, 2017). Al interior de estas reuniones dedicadas principalmente al aprendizaje se generan encuentros sociales y se promueve la socialización de los niños con la comunidad.

Reavis \& Zakriski (2005) encontraron que las reducidas interacciones que experimentan los menores expuestos al modelo Homeschooling con otros niños, origina que sean muy dependientes de la amistad que entablan con otros infantes. Ello debido a que buscan mantener el apoyo social y el bienestar personal. Además, los niños expuestos al modelo Homeschooling no eran capaces de reconocer la diversidad de aprendizajes que se pueden obtener del entorno social, cuando tratan únicamente con compañeros habituales de juego. La escasa diversidad de los contextos de interacción puede provocar que ellos evidencien dificultades para reconocer la variedad de situaciones comunicativas y sociales que se pueden presentar en diferentes contextos.

En contraste con lo anterior, Rahma et al. (2018) afirman que aspectos como la comunicación, el juego, la empatía se desarrolla suficientemente en los niños expuestos al modelo Homeschooling. Lo anterior debido a que pueden establecer vínculos sociales positivos que los ayuda a mantener relaciones de calidad. Estos autores argumentan que las relaciones sociales de los niños que estudian en el hogar tienden a ser escasas, pero representan amistades mucho más íntimas y con mayor cercanía, porque comparten tanto el tiempo como las actividades que realizan. Adicionalmente, se reportan actitudes favorables hacia los educadores, relaciones más auténticas con los padres y una autoestima más alta (Kingston \& Medlin, 2005).

En otro estudio de carácter comparativo, se encontró que los niños educados en el hogar informan más lazos afectivos con sus amigos cercanos que los que reciben educación pública (McKinley et al., 2007). Por este motivo, McKinley concluye que tanto la calidad de la amistad como la cercanía están relacionadas con la autoestima y la sociabilidad. También, afirma que estos factores pueden proteger a los niños de sentimientos y sensaciones negativos como son la depresión y la ansiedad. En la educación privada, Reavis \& Zakriski (2005) comparan niños pertenecientes a este sistema tradicional con niños educados en casa. Reportan que, en promedio, ambos grupos de niños tienen la misma cantidad de amigos cercanos y la calidad de la amistad es similar. Sin embargo, en el grupo de niños del modelo Homeschooling, aquellos que tenían amistades de baja calidad presentaban más ansiedad e impotencia que los niños del modelo Homeschooling con amistades de alta calidad Reavis \& Zakriski (2005). Estos hallazgos sugieren que el contexto social de su educación puede implicar tanto beneficios como riesgos para los niños expuestos al modelo Homeschooling. Los autores sugieren que los niños expuestos a este modelo parecen ser más vulnerables a la angustia psicológica en ausencia de una amistad de alta calidad.

Por otra parte, se describe que un aspecto determinante de la interacción social de los niños en esta modalidad se relaciona con el nivel de formación de los docentes. Lo anterior debido a que no se supervisa la calidad de la educación que imparten a los niños y si incluyen el desarrollo del componente social. Esto podría influir en la calidad y cantidad de oportunidades para desarrollar la socialización en los niños (McKinley et al., 2007). 
Con relación a los padres, se encontró que cuanto mayor es el apego de la madre, menores son los encuentros sociales del niño (Guterman \& Neuman, 2017). Ello debido a que las personalidades de los padres y la forma en que se practica la educación en el hogar se correlacionan significativamente con sus encuentros sociales.

Otra perspectiva frente a la interacción social es brindada por Aliaga Castillo (2017), quien asegura que en Chile las familias son muy explícitas para resaltar que uno de los puntos fuertes de la educación en el hogar es precisamente el desarrollo de un excelente nivel de sociabilidad.

\section{Cognición social}

De acuerdo con Medlin (2006), los niños educados en el hogar se describieron a sí mismos como más cooperativos, asertivos, empáticos y con mayor autocontrol que los niños de las escuelas públicas. Lo anterior se complementa con lo reportado en el estudio de Kingston \& Medlin (2005). En este trabajo se compararon dos grupos: niños expuestos al modelo Homeschooling y niños de escuelas públicas. Es destacable que, aunque los puntajes de ambos grupos relacionados con la empatía no tenían diferencias significativas, las niñas eran más empáticas (tanto en el grupo del Homeschooling como en el grupo de escuelas públicas) que los niños. Ello se demostraba en que las niñas estaban más dispuestas a tomar la perspectiva de los demás y tenían más probabilidades de sentir preocupación y compasión por los otros. Así mismo, se encontró que los niños que eran más empáticos también eran más altruistas. A su vez, los niños educados en casa eran considerablemente más altruistas que los de la escuela pública. Estos resultados podrían relacionarse con el hecho de que los niños educados en el hogar traducen más fácilmente los pensamientos y sentimientos empáticos en intenciones altruistas que los niños de las escuelas públicas (Kingston \& Medlin, 2005).

\section{Pragmática: Comunicación verbal y no verbal}

En los estudios no se encontró información que incluyera a los actos de habla, las intenciones comunicativas, la prosodia, las máximas conversacionales y el discurso. Al no contar con información sobre el discurso, tampoco se obtuvo evidencia sobre aspectos como la asertividad, el mantenimiento del tópico conversacional, la reciprocidad social, la cohesión y coherencia.

\section{Procesamiento del lenguaje expresivo y comprensivo}

En la búsqueda realizada no hubo resultados referidos a la comprensión y expresión del lenguaje oral y escrito. Por lo tanto, no se obtuvo información sobre la fonología, la morfología, la sintaxis y la semántica.

\section{DISCUSIÓN}

$\mathrm{Al}$ analizar la relación existente entre la educación en el hogar o Homeschooling y el desarrollo de la comunicación social es posible afirmar que la mayoría de los estudios muestran que la educación en el hogar impacta de manera positiva en el desarrollo de los componentes de interacción y cognición social. Los niños expuestos al modelo Homeschooling desarrollan mejor estos componentes que los escolares que asisten a una escuela tradicional (Kingston \& Medlin, 2005; Medlin, 2006) o al menos su desarrollo es similar al esperado para los niños de su edad (Aliaga Castillo, 2017; Beck, 2008, 2010; Reavis \& Zakriski, 2005). En la encuesta acerca del modelo Homeschooling que se realizó en Chile se señala que la ausencia de sociabilización no es un problema (Aliaga Castillo, 2017). Por el contrario, los padres indican múltiples oportunidades de sociabilización con sus hijos. Además, los niños educados en el hogar muestran mejores habilidades para relacionarse con otros sujetos, porque no tienen adquiridas respuestas estándares enseñadas por el sistema educativo tradicional. Por ejemplo, no se le enfatiza el respeto a la autoridad, tampoco se destacan los límites entre pares o entre personas de diferentes edades, ni se establece lo que un niño puede y/o debe saber a ciertas edades.

Sin embargo, algunos estudios se oponen a estos hallazgos y mencionan diferencias en las habilidades sociales de los niños expuestos al Homeschooling. Se concluye que los niños con educación privada son mejores en términos de cooperación, afirmación, autocontrol y habilidades sociales generales que los niños educados en casa (McKinley et al., 2007). Además, se indica que el desarrollo de las habilidades socioemocionales se abordará mejor en el currículo escolar bajo la guía de docentes competentes y con recursos didácticos adecuados (Rahma et al., 2018).

Al cotejar estas discrepancias se encuentra que uno de los factores diferenciales puede estar en la relación de los padres con los niños educados en el hogar (Guterman \& Neuman, 2017). Por lo tanto, este postulado estaría relacionado con otros estudios que destacan el papel de la familia en las habilidades sociales de los niños. Por ejemplo, se encontró que las familias con mejores espacios de comunicación, expresiones de afecto y un manejo de normas claras, son generadoras de un repertorio amplio de habilidades sociales; mientras que aquellas que poseen una estructura disciplinada, caracterizada por acciones autoritarias de los padres, 
se asocian a un insuficiente nivel de desempeño social (IsazaValencia \& Henao-López, 2011). Debido a lo anterior, es posible que los hijos desarrollen mejores habilidades sociales, pues sus padres procuran mantener una relación positiva y recíproca con los niños, tienen un conocimiento íntimo de sus necesidades particulares y poseen un gran interés por su bienestar.

Es necesario resaltar que Guterman \& Neuman (2017) encontraron que los padres con pocos hijos sienten una mayor necesidad de generar en ellos relaciones sociales. Es decir, la tendencia de los padres por establecer relaciones sociales se expresará más en familias con menos hijos, ya que la necesidad del establecimiento de relaciones sociales por fuera de la familia es muy importante desde el punto de vista de los padres (Beck, 2008).

A pesar de la importancia de los padres en el desarrollo de las habilidades sociales, es necesario que ellos no asuman cabalmente el rol de educadores, dado que puede ser un factor de riesgo en la salud mental de los progenitores. Al respecto, Baker (2019) afirma que el trabajo adicional puede provocar un aumento en los niveles de estrés en las madres al exigir que utilicen una gran cantidad de recursos como tiempo, energía, trabajo emocional y dinero en beneficio para sus hijos. Por ejemplo, en la pandemia los padres que fueron responsables de administrar la educación en el hogar tenían mayores niveles de estrés, preocupación, aislamiento social y conflictos domésticos, que aquellos que no tuvieron esa responsabilidad. Incluso, algunos padres informaron que aumentaron en el uso de alcohol y/o drogas (Thorell et al., 2021).

Es importante considerar que la carencia de habilidades sociales asertivas favorece la aparición de comportamientos disfuncionales en el ámbito familiar y escolar. En la adolescencia, el papel de las habilidades sociales es fundamental para el acercamiento a pares, la conformación de la pareja y la participación en diversos grupos e influye también en la percepción que el joven tiene de sí, de los otros y de la realidad (Betina Lacunza \& Contini de González, 2011).

Respecto a la cognición social de los niños educados en el hogar, Rahma et al. (2018) exponen que la empatía se desarrolla lo suficiente de acuerdo con lo esperado para la edad. Así, el juego se transforma en la principal herramienta de aprendizaje sobre la amistad, y sobre aspectos relacionados con la cooperación y la competencia; sin embargo, este hallazgo podría cuestionarse al ser un estudio de carácter descriptivo y no experimental. Desde otra perspectiva, McKinley et al. (2007) afirman que la heterogeneidad en los resultados depende del contexto y las condiciones en donde sucede la interacción social. Por ello, se deberá analizar la cooperación, la afirmación, el autocontrol y las habilidades sociales generales. Así, los niños con educación privada obtuvieron puntajes significativamente más altos que los niños con educación en el hogar (McKinley et al., 2007), lo que plantea que la distinción no sólo se produce en el modelo de educación sino en el mismo sistema.

Es destacable que no se encuentran resultados respecto al desarrollo de habilidades pragmáticas y de procesamiento de lenguaje en los niños expuestos a la educación en el hogar. Lo anterior limita el alcance de la revisión y el análisis e interpretación de los resultados obtenidos de manera holística.

Obtener información sobre estos ámbitos permitiría tener un panorama más amplio y una mayor comprensión del desarrollo de la comunicación social en niños expuestos a este sistema educativo. Ello debido a que la pragmática y el procesamiento del lenguaje permiten usar los recursos para interactuar y cumplir sus propósitos comunicativos.

En nuevas investigaciones será importante ampliar este panorama, diferenciando aspectos propios del desarrollo de la comunicación social. Por ejemplo, en la edad preescolar debe centrarse en la obtención de la intención comunicativa a través de métodos naturalistas como parte de una evaluación general de las habilidades de comunicación social. En niños mayores es necesario realizar una investigación exhaustiva de los actos de habla, las habilidades de conversación, la narración, la comprensión de las intenciones, así como la capacidad del niño para emplear señales contextuales de comprensión (Adams, 2002).

Estos estudios deberían ser efectuados principalmente en el contexto latinoamericano, ya que la mayoría de los trabajos fueron realizados en Estados Unidos, Noruega, Indonesia e Israel. Se requiere que se considere las particularidades del sistema educativo y las diferencias y desigualdades que puedan presentarse en relación con el acceso y la calidad de la educación (Puryear, 1997). También es importante homogeneizar la población a estudiar para obtener resultados más precisos. Es necesario señalar que cuatro estudios incluyen directamente niños (McKinley et al., 2007; Medlin, 2006; Rahma et al., 2018; Reavis \& Zakriski, 2005), cuatro consideran a las familias o educadores en el hogar (Aliaga Castillo, 2017; Beck, 2008, 2010; Guterman \& Neuman, 2017) y un estudio participan ambas poblaciones (Kingston \& Medlin, 2005).

Estos aspectos influyen de manera directa en la interpretación de los resultados y enriquece el rango de ópticas por analizar, porque 
considera la diversidad de la población que forma parte de la educación en el hogar. Además, invita a reflexionar acerca de las razones para implementar este modelo educativo y la forma en la cual se lleva a cabo. Todo lo anterior dificulta determinar con precisión el impacto de esta práctica educativa en el desarrollo social y comunicativo de los niños (Cheng \& Donnelly, 2019).

Como afirma Medlin (2013) es claro que las habilidades sociales no deberían ser vistas como rasgos estáticos. Es por esta razón que dichas habilidades implican procesos dinámicos e interactivos que idealmente deberían ser evaluados en los entornos naturales y cotidianos en donde los niños educados en el hogar desarrollan su vida social.

En Latinoamérica, con la actual situación originada por el COVID-19, es necesario evaluar las acciones educativas de la educación inclusiva y estudiar las diferentes trayectorias implementadas durante la emergencia con el propósito de continuar con el servicio educativo. Se destacan trayectorias que incluyen planes, programas o proyectos nacionales de estudios en casa con modalidades de atención a distancia; la educación a distancia con una modalidad de atención virtual y la radio, la televisión, así como recursos impresos, como estrategias de trabajo para llegar a rincones donde la conectividad no es posible (Delgado-Valdivieso, 2020).

Es así como en esta época de cambios educativos asociados a la situación de pandemia por el COVID-19 se identifica la necesidad de continuar investigando al respecto y de incorporar estrategias pedagógicas del modelo Homeschooling al sistema educativo actual, teniendo en cuenta sus utilidades en el marco de esta situación mundial.

Es relevante mencionar que investigaciones recientes han identificado elementos positivos en las rutinas de los niños al pasar más tiempo leyendo y realizando actividades extraescolares en casa (Champeaux et al., 2020), lo que genera oportunidades de aprendizaje, pero a la vez necesidades respecto a las habilidades socioemocionales de los niños como la salud mental, el bienestar y el comportamiento. Es necesario considerar que el riesgo de un aumento de los problemas socioemocionales puede ser mayor para quienes viven en hogares más pobres, con menor nivel educativo y en aquellos que tienen habilidades socioemocionales más disminuidas.

En futuros estudios será importante analizar información que aborde en detalle a los componentes anteriores en población expuesta al modelo Homeschooling. Además, desde la Fonoaudiología, como disciplina que estudia la comunicación humana y sus desórdenes, se pueden orientar futuras investigaciones a población infanto-juvenil con dificultades de comunicación, en donde se aborde su escolarización y su desarrollo de los componentes de la comunicación social, ya sea desde un sistema educativo tradicional o desde la educación en casa. El fonoaudiólogo es uno de los profesionales idóneos para identificar y evaluar a la comunicación social, además de diagnosticar y tratar sus posibles trastornos. Así, se espera que los resultados de la evaluación permitan caracterizar la comunicación social de esta población y determinar sus necesidades comunicativas. A partir de la información obtenida, en los casos que corresponda, se diseñarán estrategias dirigidas a padres, educadores, cuidadores e incluso a los propios niños. Dichas estrategias buscan impactar de manera importante en el favorecimiento de las habilidades comunicativas y sociales de los menores. Todo ello con el propósito de prevenir dificultades que puedan repercutir de manera desfavorable en los componentes de la comunicación social. Asimismo, implementar, desde una perspectiva fonoaudiológica, estrategias adicionales para promover la comunicación, la interacción social y el desarrollo de habilidades socioemocionales, involucrando el uso de recursos tecnológicos e innovadores.

\section{Limitaciones}

Se pueden reconocer tres grandes limitaciones del estudio. La primera refiere al número de artículos, la segunda a la heterogeneidad en las metodologías presentadas en cada estudio y la tercera se relaciona con la sistematización de los resultados de la información. La dos primeras limitaciones no permiten generalizar los resultados a diferentes poblaciones. Por su parte, la tercera implica que la investigación está expuesta a varios sesgos, por lo que sería necesario plantear una revisión sistemática a futuro, que incorpore varios de los estudios realizados durante la pandemia.

\section{CONCLUSIONES}

La mayoría de los estudios muestran un impacto positivo de la educación en el hogar en el desarrollo de los componentes de interacción social, establecimiento de vínculos afectivos y cognición social. Los hallazgos encontrados están principalmente en el componente de la interacción social, que es uno de los cuatro factores señalados por Adams (2005). En dicho factor el desempeño en la interacción social entre pares se asocia a las actividades que puedan desarrollarse en el contexto, y con los adultos como educadores, donde se pueden generar relaciones más positivas. 
Por otro lado, en cognición social, Medlin (2006) propone que los niños expuestos al modelo Homeschooling desarrollan mayor empatía, la que también se asocia a un aumento en las intenciones altruistas de los niños (Kingston \& Medlin, 2005). En relación con las habilidades pragmáticas y de procesamiento de lenguaje no se encontró información relevante.

A lo largo de la revisión, un común denominador es que los padres o educadores en el hogar poseen una influencia significativa en la interacción social de los niños que pertenecen al modelo Homeschooling, en contraste con los niños de su edad que asisten al sistema educativo tradicional. Los niños educados en el hogar no tienen en su cotidianidad un contexto en el que se reúnan con otros niños, es por esta razón que los componentes de la comunicación social de un niño expuesto al modelo Homeschooling se convierten en un eje fundamental en su desarrollo. En general, los resultados encontrados evidencian la necesidad de ampliar la investigación en esta área, especialmente debido a la pandemia por el COVID-19, ya que en este periodo se han adaptado muchas de las estrategias del modelo Homeschooling a la educación actual de los niños y adolescentes. Finalmente, es importante reconocer el rol educativo de los fonoaudiólogos a la hora de plantear estrategias de promoción del bienestar comunicativo en niños que participan en el modelo educativo del Homeschooling.

\section{REFERENCIAS}

Acuña, X., \& Sentis, F. (2004). Desarrollo pragmático en el habla infantil. Onomázein, $10(2)$, $33-56$.

http://onomazein.letras.uc.cl/Articulos/10/2_Acuna.pdf

Adams, C. (2002). Practitioner Review: The assessment of language pragmatics. Journal of Child Psychology and Psychiatry, 43(8), 973-987. https://doi.org/10.1111/1469-7610.00226

Adams, C. (2005). Social Communication Intervention for School-Age Children: Rationale and Description. Seminars in Speech and Language, 26(03), 181-188. https://doi.org/10.1055/s-2005-917123

Adolphs, R. (2001). The neurobiology of social cognition. Current Opinion in Neurobiology, 11(2), 231-239. https://doi.org/10.1016/S0959-4388(00)00202-6

Aliaga Castillo, L. (2017). Educación en el hogar en Chile. Informe de resultados de la Encuesta Nacional. Educación, 26(50), 7-27. https://doi.org/10.18800/educacion.201701.001

Álvarez, A., \& del Río, P. (1990). Educación y Desarrollo: La Teoria De Vygotsky Y La Zona De Desarrollo Próximo. En C. Coll, J. Palacios, \& A. Marchesi (Eds.), Desarrollo psicológico y educación. II. Psicología de la Educación (pp. 93-119). Alianza Editorial. https://www.researchgate.net/profile/AmeliaAlvarez/publication/348559478_Aprendizaje_y_desarrollo_La_teoria_de_la_acti vidad_y_la_ZDP/links/60049afa45851553a05072dc/Aprendizaje-y-desarrollo-
La-teoria-de-la-actividad-y-la-ZDP.pdf

American Speech-Language-Hearing Association [ASHA]. (s. f.-a). Components of Social Communication. American Speech-Language-Hearing Association; American Speech-Language-Hearing Association. Recuperado 26 de octubre de 2021, de https://www.asha.org/practice-portal/clinical-topics/socialcommunication-disorder/components-of-social-communication/

American Speech-Language-Hearing Association [ASHA]. (s. f.-b). Social Communication. American Speech-Language-Hearing Association; American Speech-Language-Hearing Association. Recuperado 26 de octubre de 2021, de https:/www.asha.org/public/speech/development/social-communication/

Arksey, H., \& O’Malley, L. (2005). Scoping studies: Towards a methodological framework. International Journal of Social Research Methodology, 8(1), 19-32. https://doi.org/10.1080/1364557032000119616

Baker, E. E. (2019). Motherhood, homeschooling, and mental health. Sociology Compass, 13(9), e12725. https://doi.org/10.1111/soc4.12725

Bases cognitivas de la conducta social humana. (2003). II Congreso Internacional de Neuropsicología.

Beck, C. (2008). Home Education and Social Integration. En International Perspectives Home Education (pp. 87-98). https://doi.org/10.1057/9781137446855_7

Beck, C. (2010). Home education: The social motivation. International Electronic Journal of Elementary Education, 3(1), 71-81. https://files.eric.ed.gov/fulltext/EJ1052484.pdf

Benabent, J. L. (2015). La patria potestad de los padres en torno al aprendizaje en familia: El Homeschooling [Tesis de Licenciatura, Universidad Miguel Hernández de Elche]. http://dspace.umh.es/bitstream/11000/2240/1/Benabent\%20Jimeno\%20Jos\%C3 $\%$ A9\%20Lu\%C3\%ADs.pdf

Betina Lacunza, A., \& Contini de González, N. (2011). Las habilidades sociales en niños y adolescentes. Su importancia en la prevención de trastornos psicopatológicos. Fundamentos en Humanidades, 12(23), 159-182. https://www.redalyc.org/pdf/184/18424417009.pdf

Bredikyte, M., \& Hakkarainen, P. (2007). Cultural development of the child through narrative learning. Acción, pensamiento y lenguaje.

Bretherton, I., \& Beegly, M. (1982). Talking about internal states: The acquisition of an explicit theory of mind. - PsycNET. Developmental Psychology, 18(6), 906921. https://doi.org/10.1037/0012-1649.18.6.906

Cabo-González, C. (2012). El homeschooling en España: Descripción y análisis del fenómeno [Tesis Doctoral, Universidad de Oviedo]. https://www.tesisenred.net/bitstream/handle/10803/94200/UOV00100TCCG.pdf ?sequence $=5 \&$ is Allowed $=\mathrm{y}$

Champeaux, H., Mangiavacchi, L., Marchetta, F., \& Piccoli, L. (2020). Learning at home: Home schooling resources and child development during the COVID-19 lockdown. Associazione Italiana Economisti Del Lavoro, 32. https://www.aiel.it/cms/cms-files/submission/all20200904120231.pdf

Chaves Salas, A. L. (2001). Implicaciones educativas de la teoría sociocultural de Vigotsky. Revista Educación, 25(2), 59-65. https://www.redalyc.org/articulo.oa? $\mathrm{id}=44025206$ 
Cheng, A., \& Donnelly, M. (2019). New Frontiers in Research and Practice on Homeschooling. Peabody Journal of Education, 94(3), 259-262. https://doi.org/10.1080/0161956X.2019.1617576

Cicchetti, D., \& Carlson, V. (Eds.). (1989). Child Maltreatment: Theory and Research on the Causes and Consequences of Child Abuse and Neglect. Cambridge University Press. https://doi.org/10.1017/CBO9780511665707

Delgado-Valdivieso, K. (2020). Educación inclusiva durante la emergencia: Acciones en América Latina. CienciAmérica: Revista de divulgación científica de la Universidad Tecnológica Indoamérica, 9(Extra 2), 154-165. https://dialnet.unirioja.es/servlet/articulo?codigo $=7746426$

Denworth, L. (2018, abril 19). Social communication in autism, explained [Informativa]. Spectrum | Autism Research News. https://www.spectrumnews.org/news/social-communication-autism-explained/

Diaz Favier, E. (2004). Características de la comunicación en la actividad del niño de sexto año de vida. EduSol, 4(8), 14-22. https://dialnet.unirioja.es/servlet/articulo? codigo $=6111050$

Dongil-Collado, E., \& Cano-Vindel, A. (2014). Habilidades Sociales (p. 60). Sociedad Española para el estudio de la Ansiedad y el Estrés (SEAS). https://bemocion.sanidad.gob.es/comoEncontrarmeMejor/guiasAutoayuda/docs/ guia_habilidades_sociales.pdf

Elkonin, D. (1980). Psicología del juego (Pablo del Río). A. Machado Libros. https://books.google.cl/books?id=ue2NPQAACAAJ\&dq=Psicolog\%C3\%ADa + d el + juego\&hl=es\&sa $=$ X\&redir_esc $=y$

Flores, D. (2010). La escuela como agente de socialización en los niños [Tesis de Licenciatura, Universidad de Cuenca]. http://dspace.ucuenca.edu.ec/jspui/bitstream/123456789/2349/1/tps647.pdf

Ford, M. E. (1982). Social cognition and social competence in adolescence. Developmental Psychology, 18(3), 323-340. https://psycnet.apa.org/doi/10.1037/0012-1649.18.3.323

Garcia-Winner, M. (2011). Social Thinking Across the Home and School Day. YAI Autism Conferences, New York.

Guralnick, M. J. (1990). Social Competence and Early Intervention. Journal of Early Intervention, 14(1), 3-14. https://doi.org/10.1177/105381519001400101

Guterman, O., \& Neuman, A. (2017). The Role of Family and Parental Characteristics in the Scope of Social Encounters of Children in Homeschooling. Journal of Child and Family Studies, 26(10), 2782-2789. https://doi.org/10.1007/s10826-017-0773-x

Hadley, P. A., \& Schuele, C. M. (1995). Come buddy, help, help me! Adults' facilitation of peer interaction in a preschool language intervention classroom. En M. L. Rice \& K. A. Wilcox (Eds.), Building a Language-Focused Curriculum for the Preschool Classroom: A Foundation for Lifelong Communication (Vol. 1, pp. 105-125). Brookes Publishing.

Hovland, C. I. (1948). Social Communication. Proceedings of the American Philosophical Society, 92(5), 371-375. https://www.jstor.org/stable/3143048

Isaza-Valencia, L., \& Henao-López, G. (2011). Relaciones entre el clima social familiar y el desempeño en habilidades sociales en niños y niñas entre dos y tres años de edad. Acta Colombiana de Psicología, 14(1), 19-30. https://repository.ucatolica.edu.co/jspui/bitstream/10983/137/1/v14n1a03.pdf
Izaryk, K., \& Skarakis-Doyle, E. (2017). Using the Delphi Technique to Explore Complex Concepts in Speech-Language Pathology: An Illustrative Example From Children's Social Communication. American Journal of Speech-Language Pathology, 26(4), 1225-1235. https://doi.org/10.1044/2017_AJSLP-16-0046

Kingston, S., \& Medlin, R. (2005). Empathy, Altruism, and Moral Development in Home Schooled Children. Home School Researcher, 16(4), 10. https://files.eric.ed.gov/fulltext/ED573484.pdf

Landa, R. J. (2005). Assessment of social communication skills in preschoolers. Mental Retardation and Developmental Disabilities Research Reviews, 11(3), 247-252. https://doi.org/10.1002/mrdd.20079

Lebeda, S. (2007). Homeschooling: Depriving Children of Social Development The Journal of Contemporary Legal Issues, 16(99), 99-104. https://docshare.tips/development_581f6653b6d87f6c7b8b46b8.html

Luebke, R. (1999). Homeschooling in Wisconsin: A Review of Current Issues and Trends. Report. Wisconsin Policy Research Report, 12(4), 35 https://eric.ed.gov/?id=ED447571

McKinley, M. J., Asaro, J. N., Bergin, J., D’Auria, N., \& Gagnon, K. E. (2007). Social Skills and Satisfaction with Social Relationships in Home-Schooled, Private-Schooled, and Public-Schooled Children. Home School Researcher, 17(3), 6. https://files.eric.ed.gov/fulltext/ED573489.pdf

Medlin, R. (2006). Homeschooled Children's Social Skills. Social Skills, 17(1), 8. https://files.eric.ed.gov/fulltext/ED573486.pdf

Medlin, R. (2013). Homeschooling and the Question of Socialization Revisited. Peabody Journal of Education, 88(3), 284-297. https://doi.org/10.1080/0161956X.2013.796825

Orpinas, P. (2010). Social Competence. En I. B. Weiner \& W. E. Craighead (Eds.), The Corsini Encyclopedia of Psychology (pp. 1-2). American Cancer Society. https://doi.org/10.1002/9780470479216.corpsy0887

Owens, R. (2011). Desarrollo del lenguaje. Pearson-Prentice Hall.

Pelphrey, K., Adolphs, R., \& Morris, J. P. (2004). Neuroanatomical substrates of social cognition dysfunction in autism. Mental Retardation and Developmental Disabilities Research Reviews, 10(4), 259-271. https://doi.org/10.1002/mrdd.20040

Pereira Del Prette, Z., \& Del Prette, A. (2003). Psicologia de las habilidades sociales: Terapia y educación. Revista Evaluar, 3(1). https://doi.org/10.35670/1667-4545.v3.n1.609

Puryear, J. (1997). La educación en América Latina: Problemas y desafíos. Programa de promoción de la Reforma Educativa en Amética Latina y el Caribe, 7, 18. http://biblioteca.utec.edu.sv/siab/virtual/interactiva/980000016.pdf

Rahma, R., Lestari, G., \& Nugroho, R. (2018). The Social Emotional Development of Homeschooling Children. Journal of Nonformal Education, 4(2), 151-160. https://doi.org/10.15294/JNE.V4I2.15975

Reavis, R., \& Zakriski, A. (2005). Are home-schooled children socially at-risk or socially protected? The Brown University Child and Adolescent Behavior Letter, 21(9), 4-5. https://www.researchgate.net/publication/333455284_Are_homeschooled_children_socially_at-risk_or_socially_protected/citations

Red En Familia. (2012). Preguntas frecuentes - EnFamilia. Preguntas frecuentes. http://www.enfamilia.co/acogida/ 
Rizo García, M. (2006). La interacción y la comunicación desde los enfoques de la psicología social y la sociología fenomenológica. Breve exploración teórica. Anàlisi: quaderns de comunicació i cultura, 2(33), 45-62. https://raco.cat/index.php/Analisi/article/view/51739

Run, C. (2016). Conoce las ventajas y desventajas del Homeschooling. https://www.universia.net/ec/actualidad/orientacion-academica/conoce-ventajasdesventajas-homeschooling-1137202.html

Semrud-Clikeman, M. (2007). Social Competence in Children. Springer US. https://doi.org/10.1007/978-0-387-71366-3 1

Serra, M. (2013). Comunicación y lenguaje. La nueva neuropsicología cognitiva, I (Edicions Universitat Barcelona). Edicions Universitat Barcelona. https://books.google.com.co/books/about/Comunicaci\%C3\%B3n_y_lenguaje_La _nueva_neurop.html?id=d3CwBAAAQBAJ\&redir_esc $=y$

Shatz, M., \& Gelman, R. (1973). The Development of Communication Skills: Modifications in the Speech of Young Children as a Function of Listener. Monographs of the Society for Research in Child Development, 38(5), 1-38. https://doi.org/10.2307/1165783

Solovieva, Y., \& Quintanar, L. (2012). La actividad de juego en la edad preescolar. Trillas. https://www.curriculumnacional.cl/portal/Autor/YuliaQuintanar-Soloviera/72304:La-actividad-de-juego-en-la-edad-preescolar
Stainton, R. (2000). Perspectives in the Philosophy of Language: A Concise Anthology (Broadview Press). Broadview Press.

Thorell, L., Skoglund, C., de la Peña, A. G., Baeyens, D., Fuermaier, A. B. M., Groom, M. J., Mammarella, I. C., van der Oord, S., van den Hoofdakker, B. J., Luman, M., de Miranda, D. M., Siu, A. F. Y., Steinmayr, R., Idrees, I., Soares, L. S., Sörlin, M., Luque, J. L., Moscardino, U. M., Roch, M., ... Christiansen, H. (2021). Parental experiences of homeschooling during the COVID-19 pandemic: Differences between seven European countries and between children with and without mental health conditions. European Child \& Adolescent Psychiatry. https://doi.org/10.1007/s00787-020-01706-1

Topping, K., Bremner, W., \& Holmes, E. (2000). Social competence: The social construction of the concept. En R. Bar-On \& D. A. Parker (Eds.), The handbook of emotional intelligence: Theory, development, assessment, and application at home, school, and in the workplace (pp. 28-39). Jossey-Bass.

Valle-Aparicio, J. E. (2012). Enseñar en casa o en la escuela. La doctrina legal sobre el homeschooling en España. Perfiles Educativos, 34(138), Article 138. https://doi.org/10.22201/iisue.24486167e.2012.138.34161

Vigotsky, L. (1977). Pensamiento y lenguaje. Paidos. https://www.planetadelibros.cl/libro-pensamiento-y-lenguaje/129679

Vigotsky, L. (1984). El instrumento y el signo en el desarrollo del niño. Pedagogía. 


\section{ANEXO}

Tabla 1. Descripción de artículos seleccionados.

\begin{tabular}{|c|c|c|c|c|c|c|}
\hline País & $\begin{array}{l}\text { Autor (es), } \\
\text { (año) }\end{array}$ & Título & Objetivo & Metodología & Participantes & Resultados \\
\hline \multirow[t]{4}{*}{$\begin{array}{l}\text { Estados } \\
\text { Unidos }\end{array}$} & $\begin{array}{l}\text { Kingston } \\
\& \text { Medlin } \\
(2005)\end{array}$ & $\begin{array}{l}\text { Empathy, Altruism, and } \\
\text { Moral Development in } \\
\text { Home Schooled } \\
\text { Children. }\end{array}$ & $\begin{array}{l}\text { Comparar la empatía, el } \\
\text { altruismo, el razonamiento } \\
\text { moral y el comportamiento } \\
\text { prosocial en niños educados } \\
\text { en el hogar y niños que } \\
\text { asisten a escuelas públicas. }\end{array}$ & $\begin{array}{l}\text { Cuantitativo } \\
\text { descriptivo. }\end{array}$ & $\begin{array}{l}\text { El grupo de escuela pública } \\
\text { tuvo } 80 \text { niños entre tercer y } \\
\text { quinto grado y el grupo } \\
\text { homeschooling tuvo } 30 \\
\text { niños. Los padres también } \\
\text { participaron en el estudio. }\end{array}$ & $\begin{array}{l}\text { Los niños educados en el hogar } \\
\text { traducen más fácilmente los } \\
\text { pensamientos y sentimientos } \\
\text { empáticos en intenciones } \\
\text { altruistas que los niños de las } \\
\text { escuelas públicas. }\end{array}$ \\
\hline & $\begin{array}{l}\text { McKinley } \\
\text { et al. } \\
(2007)\end{array}$ & $\begin{array}{l}\text { Social Skills and } \\
\text { Satisfaction with Social } \\
\text { Relationships in Home- } \\
\text { Schooled, Private- } \\
\text { Schooled, and Public- } \\
\text { Schooled Children. }\end{array}$ & $\begin{array}{l}\text { Comparar las habilidades } \\
\text { sociales de niños con } \\
\text { educación en el hogar, con } \\
\text { educación privada y niños } \\
\text { con educación pública. }\end{array}$ & $\begin{array}{l}\text { Cuantitativo } \\
\text { descriptivo. }\end{array}$ & $\begin{array}{l}53 \text { niños con educación en } \\
\text { el hogar, } 49 \text { con educación } \\
\text { privada y } 48 \text { niños con } \\
\text { educación pública entre las } \\
\text { edades de } 8 \text { y } 12 \text { años. }\end{array}$ & $\begin{array}{l}\text { Los niños con educación privada } \\
\text { obtuvieron puntajes } \\
\text { significativamente más altos que } \\
\text { los niños con educación en el } \\
\text { hogar en medidas de cooperación, } \\
\text { afirmación, autocontrol y } \\
\text { habilidades sociales generales. }\end{array}$ \\
\hline & $\begin{array}{l}\text { Medlin } \\
(2006)\end{array}$ & $\begin{array}{l}\text { Homeschooled } \\
\text { Children's Social Skills. }\end{array}$ & $\begin{array}{l}\text { Examinar las habilidades } \\
\text { sociales en niños educados } \\
\text { en el hogar desde su propio } \\
\text { punto de vista. }\end{array}$ & $\begin{array}{l}\text { Metodología } \\
\text { mixta. }\end{array}$ & $\begin{array}{l}70 \text { niños educados en el } \\
\text { hogar, } 32 \text { niños y } 38 \text { niñas } \\
\text { en los grados } 3 \text { a } 6 .\end{array}$ & $\begin{array}{l}\text { Los niños educados en el hogar } \\
\text { poseen mejores habilidades } \\
\text { sociales que los niños que } \\
\text { atienden escuelas tradicionales. }\end{array}$ \\
\hline & $\begin{array}{l}\text { Reavis \& } \\
\text { Zakriski } \\
(2005)\end{array}$ & $\begin{array}{l}\text { Are home-schooled } \\
\text { children socially at-risk } \\
\text { or socially protected? }\end{array}$ & $\begin{array}{l}\text { Comparar las relaciones } \\
\text { entre pares y el ajuste } \\
\text { psicológico de los niños } \\
\text { educados en el hogar (HS) } \\
\text { y tradicionalmente (TS). }\end{array}$ & $\begin{array}{l}\text { Mixto, con } \\
\text { predominio } \\
\text { cualitativo. }\end{array}$ & $\begin{array}{l}16 \text { niños educados en el } \\
\text { hogar y } 48 \text { niños } \\
\text { tradicionalmente educados. }\end{array}$ & $\begin{array}{l}\text { Las relaciones de los niños con } \\
\text { HS y TS son bastante similares. } \\
\text { En promedio, ambos grupos de } \\
\text { niños tenían la misma cantidad de } \\
\text { amigos cercanos y de calidad } \\
\text { similar. }\end{array}$ \\
\hline \multirow[t]{2}{*}{ Noruega } & $\begin{array}{l}\text { Beck } \\
(2008)\end{array}$ & $\begin{array}{l}\text { Home Education and } \\
\text { Social Integration. }\end{array}$ & $\begin{array}{l}\text { Analizar la socialización } \\
\text { entre estudiantes noruegos } \\
\text { educados en el hogar de } \\
\text { diferentes regiones. }\end{array}$ & $\begin{array}{l}\text { Cualitativo } \\
\text { descriptivo. }\end{array}$ & $\begin{array}{l}128 \text { educadores de hogar } \\
\text { noruegos. }\end{array}$ & $\begin{array}{l}\text { Entre los educadores en el hogar } \\
\text { que están registrados y } \\
\text { supervisados, la educación en el } \\
\text { hogar parece producir estudiantes } \\
\text { bien socializados. }\end{array}$ \\
\hline & $\begin{array}{l}\text { Beck } \\
(2010)\end{array}$ & $\begin{array}{l}\text { Home Education: The } \\
\text { Social Motivation. }\end{array}$ & $\begin{array}{l}\text { Indagar por los motivos } \\
\text { para implementar } \\
\text { educación real en el hogar y } \\
\text { los motivos para instaurar } \\
\text { una posible educación en el } \\
\text { hogar. }\end{array}$ & $\begin{array}{l}\text { Cualitativo } \\
\text { retrospectivo. }\end{array}$ & $\begin{array}{l}\text { Muestra de } 564 \text { padres } \\
\text { noruegos con hijos } \\
\text { estudiantes en la escuela } \\
\text { obligatoria noruega. }\end{array}$ & $\begin{array}{l}\text { Algunas experiencias escolares } \\
\text { particulares de la escuela privada } \\
\text { pueden influir en algunas áreas de } \\
\text { las habilidades sociales de los } \\
\text { niños, como mejorar su } \\
\text { cooperación, asertividad y } \\
\text { autocontrol y reduciendo su } \\
\text { soledad. }\end{array}$ \\
\hline Indonesia & $\begin{array}{l}\text { Rahma } \\
\text { et al. } \\
(2018)\end{array}$ & $\begin{array}{l}\text { The Social Emotional } \\
\text { Development of } \\
\text { Homeschooling } \\
\text { Children. }\end{array}$ & $\begin{array}{l}\text { Discutir el desarrollo social } \\
\text { y emocional de los niños } \\
\text { con educación en el hogar. }\end{array}$ & $\begin{array}{l}\text { Cualitativo } \\
\text { descriptivo. }\end{array}$ & $\begin{array}{l}\text { Estudiantes de Khoiru } \\
\text { Ummah Surabaya } \\
\text { Homeschooling }\end{array}$ & $\begin{array}{l}\text { El desarrollo de habilidades } \\
\text { socioemocionales se abordará } \\
\text { mejor en el currículo escolar bajo } \\
\text { la guía de docentes competentes y } \\
\text { con recursos didácticos } \\
\text { adecuados. }\end{array}$ \\
\hline Israel & $\begin{array}{l}\text { Guterman } \\
\& \text { Neuman } \\
(2017)\end{array}$ & $\begin{array}{l}\text { The Role of Family and } \\
\text { Parental Characteristics } \\
\text { in the Scope of Social } \\
\text { Encounters of Children } \\
\text { in Homeschooling. }\end{array}$ & $\begin{array}{l}\text { Examinar las relaciones } \\
\text { entre los encuentros } \\
\text { sociales de un niño y las } \\
\text { personalidades de los } \\
\text { padres. }\end{array}$ & $\begin{array}{l}\text { Mixto, con } \\
\text { predominio } \\
\text { cualitativo. }\end{array}$ & $\begin{array}{l}140 \text { padres que educaron en } \\
\text { casa a sus niños. }\end{array}$ & $\begin{array}{l}\text { Las personalidades de los padres } \\
\text { y la forma en que se practica la } \\
\text { educación en el hogar se } \\
\text { correlacionan significativamente } \\
\text { con los encuentros sociales de } \\
\text { niños educados en casa. }\end{array}$ \\
\hline Chile & $\begin{array}{l}\text { Aliaga } \\
\text { Castillo } \\
(2017)\end{array}$ & $\begin{array}{l}\text { Educación en el hogar } \\
\text { en Chile. Informe de } \\
\text { resultados de la } \\
\text { Encuesta Nacional. }\end{array}$ & $\begin{array}{l}\text { Indagar por los motivos que } \\
\text { tuvieron los padres, los } \\
\text { valores, que les interesa y } \\
\text { sus formas de socialización. }\end{array}$ & $\begin{array}{l}\text { Cualitativo } \\
\text { descriptivo. }\end{array}$ & $\begin{array}{l}67 \text { familias que practican el } \\
\text { homeschooling en Chile. }\end{array}$ & $\begin{array}{l}\text { Las familias indican múltiples } \\
\text { oportunidades de sociabilización } \\
\text { que los padres, en familia o } \\
\text { grupos, les brindan a sus hijos/as. }\end{array}$ \\
\hline
\end{tabular}

